\title{
Current and emerging treatment options in the management of Friedreich ataxia
}

This article was published in the following Dove Press journal:

Neuropsychiatric Disease and Treatment

30 July 2010

Number of times this article has been viewed

\section{Michelangelo Mancuso \\ Daniele Orsucci \\ Anna Choub \\ Gabriele Siciliano}

Department of Neuroscience, Neurological Clinic, University of Pisa, Pisa, Italy

Correspondence: Michelangelo Mancuso Department of Neuroscience, Neurological Clinic, University of Pisa, via Roma 67, 56 I 26 Pisa, Italy

Tel +39-050-992440

Fax +39-050-554808

Email mmancuso@inwind.it

\begin{abstract}
Friedreich ataxia (FRDA) is the most common autosomal recessive ataxia. Oxidative damage within the mitochondria seems to have a key role in the disease phenotype. Therefore, FRDA treatment options have been mostly directed at antioxidant protection against mitochondrial damage. Available evidence seems to suggest that patients with FRDA should be treated with idebenone, because it is well tolerated and may reduce cardiac hypertrophy and, at higher doses, also improve neurological function, but large controlled clinical trials are still needed. Alternatively, gene-based strategies for the treatment of FRDA may involve the development of small-molecules increasing frataxin gene transcription. Animal and human studies are strongly needed to assess whether any of the potential new treatment strategies, such as iron-chelating therapies or treatment with erythropoietin or histone deacetylase inhibitors and other gene-based strategies, may translate into an effective therapy for this devastating disorder. In this review, we try to provide an answer to some questions related to current and emerging treatment options in the management of FRDA.
\end{abstract}

Keywords: frataxin, idebenone, oxidative stress

\section{Introduction}

Friedreich ataxia (FRDA, OMIM \#229300) is the most common autosomal recessive ataxia among Caucasian population, and it is caused by mutations in the $F X N$ gene (OMIM *606829), mainly an expanded GAA triplet repeat in the intron $1 .{ }^{1}$ Age at onset is typically 5-25 years. Sensory neurons in the dorsal root ganglia are lost initially, with secondary degeneration of the spinocerebellar tract, pyramidal tract, and dorsal columns. ${ }^{2}$ FRDA is, therefore, characterized by progressive gait and limb ataxia, dysarthria, loss of vibration and proprioceptive sense, areflexia, abnormal eye movements, and pyramidal signs. Involvement of the auditory sensory neurons and pathways may also be found, as in optic atrophy. ${ }^{3}$ Ataxia of mixed cerebellar and sensory type is the cardinal symptom. The first symptom is usually gait instability, though scoliosis may already be present when neurologic symptoms appear, and, in rare cases, hypertrophic cardiomyopathy is diagnosed before the onset of ataxia.

In patients with FRDA, voxel-based morphometry showed a symmetrical volume loss in dorsal medulla, infero-medial portions of the cerebellar hemispheres, the rostral vermis, and in the dentate region. ${ }^{4}$ No volume loss in cerebral hemispheres was observed. The atrophy of the cerebellum and medulla correlated with the severity of the clinical deficit and disease duration. ${ }^{4}$ Moreover, some magnetic resonance imagingbased studies found cerebral white matter atrophy or dysfunction., 
A possible manifestation of this disease is hypertrophic cardiomyopathy, described in up to two-third of patients with FRDA. ${ }^{7}$ Ventricular arrhythmias can also occur. Later in the course of the disease, the hypertrophied heart can develop systolic dysfunction and heart failure and arrhythmias are possible causes of death in these patients. ${ }^{8}$ Diabetes, scoliosis, and pes cavus are other possible manifestations of FRDA. Clinical course is variable, but on average 10-15 years after onset, patients lose the ability to walk, stand, and sit without support. ${ }^{3}$ Age at diagnosis, which may incorporate other genetic and environmental factors, may be more important than GAA length in predicting cardiomyopathy, scoliosis, and disease progression. ${ }^{9}$

In FRDA the genetic abnormality results in the deficiency of frataxin, a protein targeted to the mitochondrion. ${ }^{10}$ In about $98 \%$ of patients, the disease is caused by a triplet GAA expansion within the first intron of the frataxin gene on chromosome 9q13, which impedes transcription of the gene and limits protein production. ${ }^{1,11}$ The repeat expansions can range from 70 to 90 repeats (normal less than 40) to over 1,000 , with inverse correlation of age at onset, severity of the disease, and associated systemic symptoms. ${ }^{2}$ Heterozygous carriers are clinically healthy. FRDA is the most common disease-causing triplet-repeat expansion identified so far, about 1 in 100 Europeans being a carrier. No other disease has been recognized to date to be caused by an expansion of GAA triplets. ${ }^{3}$ Some patients are compound heterozygotes with the GAA expansion in one allele and one of a variety of point mutations in the other allele. ${ }^{2}$ The FRDA-associated expansion shows instability when transmitted from parent to child. Expansions and contractions can both be observed and are equally likely after maternal transmission, whereas contractions are most common after paternal transmission. ${ }^{3}$ In this regard, FRDA resembles the other diseases associated with very large expansions in noncoding regions, including fragile $\mathrm{X}$ syndrome and myotonic dystrophy, and differs from the diseases that are caused by CAG repeats in coding regions, such as dominant ataxias and Huntington disease, in which size increases typically occur after paternal transmission. ${ }^{3}$

\section{FRDA pathogenetic theories and their relevance for therapeutic approaches}

Although the exact physiological function of frataxin is not known, its involvement in iron-sulfur cluster biogenesis has been suggested. ${ }^{12}$ Frataxin iron-binding capacity is quite robust. Even when 5 of the most conserved residues from the putative iron-binding region are altered, at least 2 iron atoms per monomer can be bound. ${ }^{13}$ Current evidence suggests that loss of frataxin impairs mitochondrial iron handling ${ }^{14}$ and respiratory chain function and contributes to increased oxidative stress and cellular damage. ${ }^{2}$

In a conditional knockout mouse model where frataxin was removed from the heart, transferrin receptor-1 was upregulated, resulting in increased iron uptake from transferrin. ${ }^{15}$ There is also marked downregulation of ferritin that is required for iron storage and decreased expression of the iron exporter, ferroportin 1, leading to decreased cellular iron efflux. The increased mitochondrial iron uptake is facilitated by upregulation of the mitochondrial iron transporter, mitoferrin $2 .{ }^{15}$ This stimulation of iron uptake probably attempts to rescue the deficit in mitochondrial iron metabolism that is due to downregulation of mitochondrial iron utilization (heme and iron-sulfur cluster synthesis and iron storage in mitochondrial ferritin). Therefore, increased mitochondrial iron uptake coupled with decreased utilization and release leads to mitochondrial iron-loading. ${ }^{16}$

Abnormalities of the neuronal cytoskeleton due to oxidative stress and increased protein glutathionylation have been also suggested to have a potential role in FRDA. ${ }^{17}$ Oxidative damage within the mitochondria seems to have a key role in the disease phenotype. ${ }^{18} \mathrm{~A}$ combined deficiency of a Krebs-cycle enzyme, aconitase, and 3 mitochondrial respiratory-chain complexes was reported in endomyocardial biopsy samples from patients with this disorder. ${ }^{19}$ All 4 enzymes share iron - sulfur clustercontaining proteins that are damaged by iron overload through generation of oxygen free radicals. ${ }^{19}$ Using phosphorus magnetic resonance spectroscopy, Lodi et $\mathrm{a}^{20}$ demonstrated a maximum rate of muscle mitochondrial adenosine triphosphate (ATP) production below the normal range in all the 12 studied FRDA patients and a strong negative correlation between mitochondrial ATP production and the number of GAA repeats in the smaller allele, suggesting that FRDA is a nuclear-encoded mitochondrial disorder. ${ }^{20}$ Moreover, Giacchetti et $\mathrm{a}^{21}$ reported an influence of the mitochondrial DNA polymorphisms on the FRDA phenotype. These authors studied 99 patients with FRDA and 48 control individuals, all from southern Italy. They found that patients belonging to the haplogroup $U$ class had a delay of 5 years in the disease onset and a lower rate of cardiomyopathy. ${ }^{21}$ Mitochondrial DNA polymerase ( $P O L G)$ CAG repeat instability has been also proposed as a predisposing factor that, in combination with environmental risk factors, may affect age of onset and FRDA progression. ${ }^{22}$ For the described reasons, FRDA treatment options have been mostly directed at antioxidant protection against mitochondrial damage. Interestingly, 
other studies demonstrated that in FRDA mitochondrial iron accumulation did not induce oxidative stress and that FRDA is a neurodegenerative disease not associated with oxidative damage. ${ }^{23}$

To date, no randomized controlled trial using antioxidants or any other pharmacological treatment has shown significant benefit on neurological symptoms associated FRDA. ${ }^{24}$ Moreover, the design of clinical trials in FRDA presents some problems, such as the lack of reliable biomarkers that correlate with clinical dysfunction and the rarity of the condition. Therefore, therapeutic strategies are still unclear. In this review, we try to provide an answer to some questions related to current and emerging treatment options in the management of FRDA. References for this review were identified by searches of PubMed until May 2010 with the term "Friedreich*"; articles especially considering potential therapeutic approaches were considered. Emphasis was placed on comprehensive reviews and original articles published after 1998. Other articles were identified through references from relevant articles. Only papers published in English were reviewed.

\section{Which pharmacological agents were effective in FRDA cellular and animal models? PPAR $\gamma$ agonists}

A recent microarray analysis of heart and skeletal muscle in a mouse model of frataxin deficiency showed molecular evidence of increased lipogenesis in skeletal muscle, and alteration of fiber-type composition in heart, consistent with insulin resistance and cardiomyopathy, respectively. ${ }^{25}$ Since the peroxisome proliferator-activated receptor gamma (PPAR $\gamma$ ) pathway is known to regulate both processes, dysregulation of this pathway may play a role in FRDA. PPAR $\gamma$ coactivator 1-alpha (PGC-1a) downregulation may contribute to the blunted antioxidant response observed in cells from FRDA patients. ${ }^{26} \mathrm{PGC}-1 \mathrm{a}$ is a transcriptional master regulator of mitochondrial biogenesis and antioxidant responses, and can be restored by agonist pioglitazione in FRDA cells, ${ }^{26}$ suggesting a potential therapeutic approach for FRDA. Moreover, Marmolino et $\mathrm{al}^{27}$ investigated the effect of another PPAR $\gamma$ agonist (Azelaoyl PAF) on the frataxin protein and mRNA expression profile in human neuroblastoma cells (SKNBE) and primary fibroblasts from skin biopsies from FRDA patients and healthy controls. Azelaoyl PAF increased both messenger RNA and protein levels of intracellular frataxin in SKNBE cells and fibroblasts from FRDA patients. ${ }^{27}$

\section{Iron chelators}

Iron chelators that target the mitochondrion have been proposed (ie, deferiprone). ${ }^{28,29}$ Adding the chelator deferiprone at clinical concentrations to inducibly frataxin-deficient HEK-293 cells resulted in chelation of mitochondrial labile iron, involved in oxidative stress. ${ }^{30}$ This led to restoration of impaired mitochondrial membrane and redox potentials, increased ATP production and oxygen consumption, and attenuation of mitochondrial DNA damage and reversal of hypersensitivity to apoptosis. ${ }^{30}$ On the other hand, a direct consequence of chelating mitochondrial free iron in various cell systems is a concentration and time-dependent loss of aconitase activity, which was shown to precede decreased cell proliferation. ${ }^{31}$ Therefore, if chelating excessive mitochondrial iron may be beneficial at some stage of the disease, attention should be paid to not fully deplete mitochondrial iron store. ${ }^{31} \mathrm{Li}$ et $\mathrm{al}^{32}$ investigated the regulation of frataxin expression by iron and reported that frataxin mRNA levels decreased significantly in multiple human cell lines treated with the iron chelator desferal. In addition, frataxin mRNA and protein levels decreased in fibroblast and lymphoblast cells derived from both normal controls and from patients with FRDA. ${ }^{32}$ Lymphoblasts and fibroblasts of FRDA patients show evidence of cytosolic iron depletion, which may occur as frataxin-deficient cells overload their mitochondria with iron, ${ }^{32}$ as already discussed. Therapeutic efforts should focus on an approach that combines iron removal from mitochondria with a treatment that increases cytosolic iron levels to maximize residual frataxin expression in FRDA patients. ${ }^{32}$

\section{Antioxidant agents}

Coenzyme Q10 has been widely used for the treatment of neurodegenerative disorders, as well as its analog idebenone, which shares an identical modified parahydroxybenzoate ring with Coenzyme Q10, but has a short carbon tail. Idebenone was cytoprotective in fibroblasts from patients with FRDA. ${ }^{33}$ Because of the role of mitochondrial oxidative damage in FRDA, Jauslin et $\mathrm{al}^{34}$ compared the efficacy of mitochondria-targeted and untargeted antioxidants derived from coenzyme Q10 and from vitamin $\mathrm{E}$ at preventing oxidative stress-induced cell death in cultured fibroblasts from FRDA patients in which glutathione synthesis was blocked. Ubiquinones have been shown to protect mitochondria from oxidative damage, but only a small proportion of externally administered ubiquinone is taken up by mitochondria. ${ }^{35}$ Conjugation of the lipophilic triphenylphosphonium cation to a ubiquinone moiety has produced a compound, MitoQ, 
which accumulates selectively into mitochondria. MitoQ passes easily through all biological membranes and, because of its positive charge, is accumulated several 100-fold within mitochondria driven by the mitochondrial membrane potential. ${ }^{35}$ The mitochondria-targeted antioxidant MitoQ was 800 -fold more potent than the untargeted analog idebenone. ${ }^{34}$ The mitochondria-targeted antioxidant MitoVit E was 350 -fold more potent than the water soluble analog. ${ }^{34}$ Therefore, targeted antioxidants may have therapeutic potential in FRDA and in other disorders involving mitochondrial oxidative damage, but have not been investigated in FRDA patients to date.

A dose-escalation trial found that idebenone was efficacious in a frataxin-deficient mouse model of FRDA. ${ }^{36}$ Low-dose idebenone (10 and $30 \mathrm{mg} / \mathrm{kg}$ per day) showed no benefit, whereas high-dose idebenone (90 mg/kg per day) delayed the cardiac disease onset, progression, and death of frataxin-deficient animals by 1 week. ${ }^{36}$

\section{Gene-based strategies}

FRDA is a loss of function disorder, therefore gene-based strategies designed to increase frataxin levels could be an ideal therapy for this disease, although there are still technological limitations to their clinical applicability. ${ }^{37}$ Viral vectors expressing frataxin partially corrected sensitivity to oxidative stress in FRDA fibroblasts. ${ }^{38,39}$ Furthermore, in mice with a localized frataxin reduction in the brainstem functional impairment could be corrected by exposure to HSV-1 vector expressing frataxin cDNA. ${ }^{40}$ Other studies reported that compounds specifically targeting the GAA repeat, such as DNA sequence-specific polyamides ${ }^{41}$ or pentamidine, ${ }^{42}$ were capable of increasing frataxin transcription. The exact mechanism by which these DNA-binding compounds increase transcription through GAA repeats still needs further characterization. ${ }^{37}$

\section{HDAC inhibitors}

Alternative gene-based strategy for the treatment of FRDA would involve the development of small-molecules increasing frataxin gene transcription. ${ }^{37}$ Expanded GAA repeats may silence frataxin expression inducing heterochromatin formation, and/or forming non B-DNA structures, such as triplex and sticky DNA, which block gene transcription. Therefore, possible treatments for FRDA may include drugs that facilitate chromatin opening, such as histone deacetylase (HDAC) inhibitors. ${ }^{37}$ Gene silencing at expanded $F X N$ alleles is accompanied by hypoacetylation of histones $\mathrm{H} 3$ and $\mathrm{H} 4$ and trimethylation of histone $\mathrm{H} 3$, observations that are consistent with a heterochromatin-mediated repression mechanism. ${ }^{43}$
Herman et $\mathrm{al}^{43}$ reported the synthesis and characterization of a class of HDAC inhibitors that reversed $F X N$ silencing in primary lymphocytes from individuals with FRDA. These molecules directly affected the histones associated with $F X N$, increasing acetylation at particular lysine residues on histones H3 and H4. One compound, BML-210, showed a significant increase in frataxin message levels by approximately 2 -fold. ${ }^{43}$ Butyric acid is another HDAC inhibitor reported to increase frataxin expression. ${ }^{44}$ Furthermore, compounds with pimelic diphenylamide basic structure were able to upregulate frataxin in cells from FRDA patients and in a mouse model. ${ }^{45}$ HDAC3 could be the likely cellular target of the pimelic diphenylamides HDAC inhibitors and the target for therapeutic intervention in FRDA. ${ }^{46}$ Although both the HDAC3 and HDAC1/2-specific compounds share a similar mechanism of inhibition of their target enzymes, only HDAC3-specific compounds increase frataxin gene expression and frataxin protein in cells. ${ }^{46}$ Rai et $\mathrm{al}^{47}$ treated KIKI mice (homozygous mice carrying a $[\mathrm{GAA}]_{230}$ repeat in the first intron of the mouse frataxin gene) with a novel HDAC inhibitor, compound 106, which substantially increases frataxin mRNA levels in cells from FRDA individuals. The treatment increased histone $\mathrm{H} 3$ and $\mathrm{H} 4$ acetylation in chromatin near the GAA repeat and restored wild-type frataxin levels in the nervous system and heart, as determined by quantitative reverse transcription polymerase chain reaction and semiquantitative western blot analysis. ${ }^{47}$ Lack of acute toxicity, normalization of frataxin levels, and of the transcription profile changes resulting from frataxin deficiency may provide support to a possible efficacy of this or related compounds in FRDA patients. ${ }^{47}$

\section{Conclusive remarks}

In conclusion, gene-based strategies (including HDAC inhibitors), the agonist pioglitazione, iron chelators that target the mitochondrion such as deferiprone, coenzyme Q10, vitamin E (especially their mitochondria-targeted forms), and idebenone were found to be effective in FRDA cellular and animal models. Clinical studies with some of these agents are reviewed in the next paragraphs. Moreover, there is some preliminary evidence that flavin adenine dinucleotide ${ }^{48}$ and hemin $^{44}$ may rescue the phenotype of frataxin deficiency in cellular and animal models, but further studies are still needed.

\section{Is there a role for idebenone and other antioxidants in FRDA patients? Idebenone}

The antioxidant idebenone is a short-chain benzoquinone derivative with a structure similar to coenzyme Q10 but with 
a more favorable pharmacokinetic profile. ${ }^{49}$ In vitro studies have shown that it acts both as an antioxidant, preventing damage to the mitochondrial membrane, and as an electron carrier, supporting mitochondrial function and ATP production. ${ }^{50}$ High doses of idebenone are safe and well tolerated in patients with FRDA.$^{49}$ The idebenone half-life was relatively consistent across dose levels (2.6-21.7 hours) ${ }^{49}$ It exhibited dose-dependent pharmacokinetics in daily doses up to 2,250 mg. ${ }^{51}$ Idebenone plasma levels are thought to correlate with central nervous system concentrations because of its ability to penetrate central nervous system. ${ }^{49}$

Schulz et a ${ }^{52}$ measured concentrations of 8-hydroxy2 -deoxyguanosine ( $8 \mathrm{OH} 2$ ' $\mathrm{dG})$, a marker of oxidative DNA damage, in urine and of dihydroxybenzoic acid (DHBA), a marker of hydroxyl radical attack, in plasma of 33 patients with FRDA. They found a 2.6-fold increase in normalized urinary $8 \mathrm{OH} 2$ 'dG but no change in plasma DHBA as compared with controls. Oral treatment with $5 \mathrm{mg} / \mathrm{kg} /$ day of idebenone for 8 weeks significantly decreased urinary $8 \mathrm{OH} 2$ 'dG concentrations. ${ }^{52}$

Idebenone is a promising drug for treatment of FRDA..$^{53}$ Early trials have demonstrated that low-dose idebenone $(5 \mathrm{mg} / \mathrm{kg}$ per day) reduced cardiac hypertrophy (as determined by echocardiography $)^{54,55}$ in the majority of patients with FRDA, with no influence upon clinical progression of the neurological disease. ${ }^{54,56}$

Recently, a randomized, placebo-controlled trial has been conducted on 48 patients with genetically confirmed FRDA. ${ }^{57}$ Treatment with higher doses of idebenone (up to $45 \mathrm{mg} / \mathrm{kg}$ ) was generally well tolerated and associated with improvement also in neurological function and activities of daily living in patients with FRDA. The degree of improvement correlated with the dose of idebenone, suggesting that higher doses may be necessary to have a beneficial effect on neurological function. ${ }^{57}$ It has been also suggested that the disease stage and patient age at which idebenone treatment is initiated may be important factors in the effectiveness of the therapy. ${ }^{58,59}$ Larger randomized trial focusing on the response to idebenone therapy of both neurological and heart symptoms are required to confirm whether an early diagnosis of FRDA can be exploited to initiate such antioxidant treatment in order to prevent the progression of this disorder.

\section{Other antioxidants}

To evaluate the long-term efficacy of a combined antioxidant and mitochondrial enhancement therapy on the bioenergetics and clinical course of 10 patients with FRDA, Hart et al ${ }^{60}$ performed an open-labeled pilot trial over 47 months with a combined coenzyme Q10 (400 mg/d) and vitamin E $(2,100 \mathrm{IU} / \mathrm{d})$ therapy. They reported a significant improvement in cardiac and skeletal muscle mitochondrial bioenergetics as assessed using phosphorus magnetic resonance spectroscopy, and heart function assessed by echocardiographic fraction shortening significantly improved. Although improved fraction shortening was reported, there was no impact upon the degree of cardiac hypertrophy evident before therapy was started ${ }^{60}$ These results must be interpreted with caution because of limited patient numbers and the absence of a placebo group.

Another pilot study investigated the potential for highdose CoQ10/vitamin E therapy to modify clinical progression in FRDA. ${ }^{61}$ Fifty patients were randomly divided into high-dose or low-dose CoQ10/vitamin E groups. At baseline, serum CoQ10 and vitamin E levels were significantly decreased in patients. ${ }^{61}$ During the trial, CoQ10 and vitamin E levels significantly increased in both groups. Serum CoQ10 level resulted to be the best predictor of a positive clinical response to CoQ10/vitamin E therapy. ${ }^{61}$

\section{Conclusive remarks}

Overall, because of the lack of controlled studies, the variable doses used and the association with other antioxidant medications, vitamin $\mathrm{E}$ has not been appropriately tested in FRDA, and no conclusions can yet be drawn about its safety and efficacy in this disorder. ${ }^{62}$ More research is needed to identify the role of vitamin $\mathrm{E}$, and of other antioxidant agents, if any, in the management of FRDA and other neurodegenerative disorders. ${ }^{63} \mathrm{~A}$ meta-analysis (which included 68 randomized trials with 232,606 healthy participants and patients with various diseases) reported that treatment with $\beta$-carotene, vitamin $A$, and vitamin $E$ may increase all-cause mortality. ${ }^{64}$ Further study of causes of mortality is needed. These findings contradicts observational studies, claiming that synthetic antioxidant supplements improve health. ${ }^{64}$ Therefore, more research is needed to establish the real safety of such compounds, including vitamin E. ${ }^{63}$

In conclusion, available evidence (Table 1) seems to suggest that patients with FRDA should be treated with idebenone, because it is well tolerated and may reduce cardiac hypertrophy and, at higher doses (up to $45 \mathrm{mg} / \mathrm{kg}$ ), also improve neurological function, but large controlled clinical trials are still needed.

\section{Other pharmacological agents could be useful in FRDA patients? Carnitine and creatine}

L-carnitine and creatine are natural compounds that may enhance cellular energy transduction. ${ }^{63} \mathrm{~A}$ placebo-controlled 


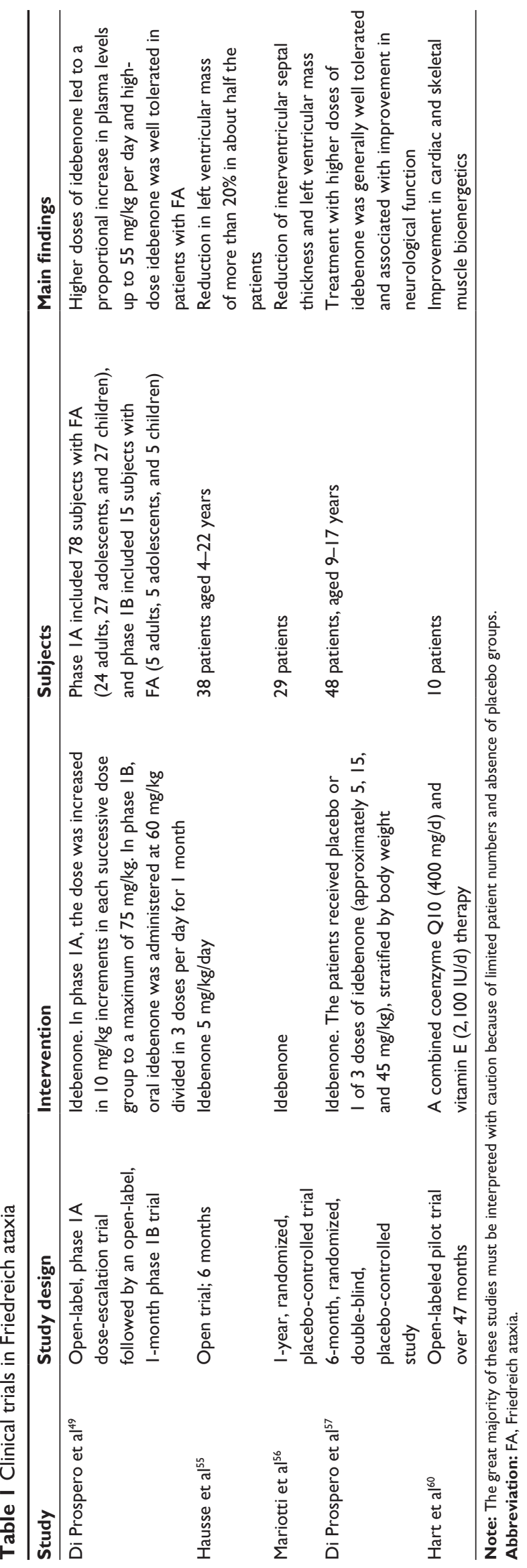

triple-phase crossover trial of L-carnitine $(3 \mathrm{~g} / \mathrm{d})$ and creatine $(6.75 \mathrm{~g} / \mathrm{d})$ has been performed in 16 patients with genetically confirmed FRDA. ${ }^{65}$ Ataxia rating scale and echocardiographic parameters remained unchanged. ${ }^{65}$

\section{Erythropoietin}

Peripherally administered erythropoietin (EPO), which could increase the amount of frataxin protein at posttranslational level in primary fibroblast cell cultures ${ }^{66}$ or isolated lymphocytes ${ }^{67}$ derived from FRDA patients, crosses the blood - brain barrier. ${ }^{68}$ It may stimulate neurogenesis, neuronal differentiation, and activate brain neurotrophic, antiapoptotic, antioxidant, and anti-inflammatory signaling. ${ }^{68}$ Boesch et $\mathrm{a}^{69}$ performed an open-label clinical pilot study with recombinant human EPO. Twelve FRDA patients received 5,000 units recombinant human EPO 3 times weekly subcutaneously. Treatment with recombinant human EPO showed a persistent and significant increase in frataxin levels after 8 weeks and a reduction of oxidative stress markers (urinary 8-hydroxydeoxyguanosine and serum peroxides). ${ }^{69}$ The same research group ${ }^{70}$ subsequently reported a 6-month open-label clinical pilot study of safety and efficacy of EPO treatment in 8 FRDA patients (2,000 units thrice a week). Scores in Ataxia Rating Scales improved significantly, with a persistent increase of frataxin levels and a reduction of oxidative stress parameters, but increases in hematocrit requiring phlebotomies occurred in 4 of 8 patients. Safety monitoring with regular blood cell counts and parameters of iron metabolism is a potential limitation of this approach, which to date has not been evaluated with a double-blind controlled study. ${ }^{70}$

\section{Conclusive remarks}

To date, pharmacological approaches other than idebenone and symptomatic therapies are not routinely indicated in patients with FRDA.

\section{Which symptomatic countermeasures are available for FRDA patients?}

Symptomatic therapy, which comprises antidiabetic therapy in case of diabetes and cardiologic therapy in case of rhythm abnormalities or heart failure, ${ }^{71}$ as well as baclofen for spasticity, ${ }^{72}$ may markedly improve quality of life and prognosis of affected individuals. The levorotatory form of 5-hydroxytryptophan could be able to modify the cerebellar symptoms in patients with FRDA, but the effect is only partial and not clinically major. ${ }^{73}$ Recent preliminary 
evidence suggests that riluzole may be potentially effective as symptomatic therapy in diverse forms of cerebellar ataxia. $^{74}$

Furthermore, in patients with cerebellar ataxia, coordinative training improved motor performance and reduced ataxia symptoms, enabling them to achieve personally meaningful goals in everyday life. ${ }^{75}$ Rehabilitation therapies usually focus on strategies and compensatory techniques for maintaining or improving abilities to continue to participate in all environmental contexts for as long as possible. ${ }^{76}$ The benefits of physical exercise programs have been demonstrated for patient with other degenerative disabilities that include ataxia, but at present there is little evidence supporting specific physical therapy interventions that would address impairments or functional concerns in patients with FRDA. ${ }^{76}$ Patients with FRDA may improve aerobic fitness by participating in stationary cycling for 20-25 minutes at $70 \%-85 \%$ of their maximum heart rate. ${ }^{77}$ The plan of care should include daily attention to range of motion, including muscle length and soft-tissue extensibility, as well as maintaining independence in mobility. ${ }^{77}$

Maintaining biomechanical alignment is another important therapeutic consideration. ${ }^{76}$ Orthopedic problems such as foot deformities and scoliosis are often treated with orthoses ${ }^{78}$ or surgery ${ }^{79}$ and may result in a temporary improvement in function. ${ }^{76}$ Early intervention for biomechanical changes in the foot significantly improves alignment and thus weight bearing ability and mobility outcomes. ${ }^{79}$

\section{Conclusion and perspectives}

In conclusion, to date the best care for patients with FRDA has not been defined according to evidence-based criteria, and all efforts should be made to obtain solid standards of care, although this goal is difficult to accomplish for a rare disease. ${ }^{80}$ The first phase 2 trial of idebenone has shown dose-dependent effects on neurological scale scores in children and adolescents with FRDA ${ }^{57}$ Large trials are needed to investigate whether all patients with FRDA can benefit from idebenone treatment, regardless of age and disease stage. ${ }^{80}$

Additional studies with animal models will be essential for an enhanced understanding of the disease pathophysiology and for the development of better therapies. ${ }^{81}$ Animal and human studies are strongly needed to assess if any of the described new treatment strategies, such as iron-chelating therapies or treatment with EPO or HDAC inhibitors and other gene-based strategies, may translate into an effective therapy for this devastating disorder.

\section{Acknowledgment}

This study was supported by Telethon Grant GUP09004.

\section{Disclosure}

The authors report no conflicts of interest in this work.

\section{References}

1. Campuzano V, Montermini L, Molto MD, et al. Friedreich's ataxia: autosomal recessive disease caused by an intronic GAA triplet repeat expansion. Science. 1996;271(5254):1423-1427.

2. Fogel BL, Perlman S. Clinical features and molecular genetics of autosomal recessive cerebellar ataxias. Lancet Neurol. 2007;6(3): 245-257.

3. Pandolfo M. Friedreich ataxia. Arch Neurol. 2008;65(10):1296-1303.

4. Della Nave R, Ginestroni A, Giannelli M, et al. Brain structural damage in Friedreich's ataxia. J Neurol Neurosurg Psychiatr. 2008;79(1): $82-85$.

5. Franca MC Jr, D'Abreu A, Yasuda CL, et al. A combined voxel-based morphometry and 1H-MRS study in patients with Friedreich's ataxia. J Neurol. 2009;256(7):1114-1120.

6. Pagani E, Ginestroni A, Della Nave R, et al. Assessment of brain white matter fiber bundle atrophy in patients with Friedreich ataxia. Radiology. 2010;255(3):882-889.

7. Hsu DT. Cardiac manifestations of neuromuscular disorders in children. Paediatr Respir Rev. 2010;11(1):35-38.

8. Kipps A, Alexander M, Colan SD, et al. The longitudinal course of cardiomyopathy in Friedreich's ataxia during childhood. Pediatr Cardiol. 2009;30(3):306-310.

9. La Pean A, Jeffries N, Grow C, Ravina B, Di Prospero NA. Predictors of progression in patients with Friedreich ataxia. Mov Disord. 2008;23(14):2026-2032.

10. Campuzano V, Montermini L, Lutz Y, et al. Frataxin is reduced in Friedreich ataxia patients and is associated with mitochondrial membranes. Hum Mol Genet. 1997;6(11):1771-1780.

11. Shishkin AA, Voineagu I, Matera R, et al. Large-scale expansions of Friedreich's ataxia GAA repeats in yeast. Mol Cell. 2009;35(1): 82-92.

12. Levi S, Rovida E. The role of iron in mitochondrial function. Biochim Biophys Acta. 2009;1790(7):629-636.

13. Correia AR, Wang T, Craig EA, Gomes CM. Iron-binding activity in yeast frataxin entails a trade off with stability in the alpha1/beta1 acidic ridge region. Biochem J. 2010;426(2):197-203.

14. Popescu BF, Pickering IJ, George GN, Nichol H. The chemical form of mitochondrial iron in Friedreich's ataxia. J Inorg Biochem. 2007;101(6):957-966.

15. Huang ML, Becker EM, Whitnall M, Rahmanto YS, Ponka P, Richardson DR. Elucidation of the mechanism of mitochondrial iron loading in Friedreich's ataxia by analysis of a mouse mutant. Proc Natl Acad Sci U S A. 2009;106(38):16381-16386.

16. Richardson DR, Huang ML, Whitnall M, Becker EM, Ponka P, Rahmanto YS. The ins and outs of mitochondrial iron-loading: the metabolic defect in Friedreich's ataxia. J Mol Med. 2010;88(4):323-329.

17. Sparaco M, Gaeta LM, Santorelli FM, et al. Friedreich's ataxia: oxidative stress and cytoskeletal abnormalities. J Neurol Sci. 2009;287(1-2):111-118.

18. Gakh O, Park S, Liu G, et al. Mitochondrial iron detoxification is a primary function of frataxin that limits oxidative damage and preserves cell longevity. Hum Mol Genet. 2006;15(3):467-479.

19. Rustin P, von Kleist-Retzow JC, Chantrel-Groussard K, Sidi D, Munnich A, Rotig A. Effect of idebenone on cardiomyopathy in Friedreich's ataxia: a preliminary study. Lancet. 1999;354(9177):477-479.

20. Lodi R, Cooper JM, Bradley JL, et al. Deficit of in vivo mitochondrial ATP production in patients with Friedreich ataxia. Proc Natl Acad Sci U S A. 1999;96(20):11492-11495. 
21. Giacchetti M, Monticelli A, De Biase I, et al. Mitochondrial DNA haplogroups influence the Friedreich's ataxia phenotype. J Med Genet. 2004;41(4):293-295.

22. Heidari MM, Houshmand M, Hosseinkhani S, Nafissi S, ScheiberMojdehkar B, Khatami M. Association between trinucleotide CAG repeats of the DNA polymerase gene (POLG) with age of onset of Iranian Friedreich's ataxia patients. Neurol Sci. 2008;29(6):489-493.

23. Seznec H, Simon D, Bouton C, et al. Friedreich ataxia: the oxidative stress paradox. Hum Mol Genet. 2005;14(4):463-474.

24. Kearney M, Orrell RW, Fahey M, Pandolfo M. Antioxidants and other pharmacological treatments for Friedreich ataxia. Cochrane Database Syst Rev. 2009;(4):CD007791.

25. Coppola G, Marmolino D, Lu D, et al. Functional genomic analysis of frataxin deficiency reveals tissue-specific alterations and identifies the PPARgamma pathway as a therapeutic target in Friedreich's ataxia. Hum Mol Genet. 2009;18(13):2452-2461.

26. Marmolino D, Manto M, Acquaviva F, et al. PGC-1alpha downregulation affects the antioxidant response in Friedreich's ataxia. PLoS One. 2010;5(4):e10025.

27. Marmolino D, Acquaviva F, Pinelli M, et al. PPAR-gamma agonist Azelaoyl PAF increases frataxin protein and mRNA expression: new implications for the Friedreich's ataxia therapy. Cerebellum. 2009;8(2):98-103.

28. Kontoghiorghes GJ, Efstathiou A, Kleanthous M, Michaelides Y, Kolnagou A. Risk/benefit assessment, advantages over other drugs and targeting methods in the use of deferiprone as a pharmaceutical antioxidant in iron loading and non iron loading conditions. Hemoglobin. 2009;33(5):386-397.

29. Richardson DR. Friedreich's ataxia: iron chelators that target the mitochondrion as a therapeutic strategy? Expert Opin Investig Drugs. 2003;12(2):235-245.

30. Kakhlon O, Manning H, Breuer W, et al. Cell functions impaired by frataxin deficiency are restored by drug-mediated iron relocation. Blood. 2008;112(13):5219-5227.

31. Goncalves S, Paupe V, Dassa EP, Rustin P. Deferiprone targets aconitase: implication for Friedreich's ataxia treatment. BMC Neurol. 2008;8:20

32. Li K, Besse EK, Ha D, Kovtunovych G, Rouault TA. Iron-dependent regulation of frataxin expression: implications for treatment of Friedreich ataxia. Hum Mol Genet. 2008;17(15):2265-2273.

33. Jauslin ML, Wirth T, Meier T, Schoumacher F. A cellular model for Friedreich ataxia reveals small-molecule glutathione peroxidase mimetics as novel treatment strategy. Hum Mol Genet. 2002;11(24):3055-3063.

34. Jauslin ML, Meier T, Smith RA, Murphy MP. Mitochondria-targeted antioxidants protect Friedreich ataxia fibroblasts from endogenous oxidative stress more effectively than untargeted antioxidants. FASEB J. 2003;17(13):1972-1974.

35. Cocheme HM, Kelso GF, James AM, et al. Mitochondrial targeting of quinones: therapeutic implications. Mitochondrion. 2007; 7 Suppl:S94-S102.

36. Seznec H, Simon D, Monassier L, et al. Idebenone delays the onset of cardiac functional alteration without correction of Fe-S enzymes deficit in a mouse model for Friedreich ataxia. Hum Mol Genet. 2004;13(10):1017-1024.

37. Hebert MD. Targeting the gene in Friedreich ataxia. Biochimie. 2008;90(8):1131-1139.

38. Fleming J, Spinoulas A, Zheng M, et al. Partial correction of sensitivity to oxidant stress in Friedreich ataxia patient fibroblasts by frataxinencoding adeno-associated virus and lentivirus vectors. Hum Gene Ther. 2005;16(8):947-956.

39. Gomez-Sebastian S, Gimenez-Cassina A, Diaz-Nido J, Lim F, WadeMartins R. Infectious delivery and expression of a $135 \mathrm{~kb}$ human FRDA genomic DNA locus complements Friedreich's ataxia deficiency in human cells. Mol Ther. 2007;15(2):248-254.

40. Lim F, Palomo GM, Mauritz C, et al. Functional recovery in a Friedreich's ataxia mouse model by frataxin gene transfer using an HSV-1 amplicon vector. Mol Ther. 2007;15(6):1072-1078.
41. Burnett R, Melander C, Puckett JW, et al. DNA sequence-specific polyamides alleviate transcription inhibition associated with long GAA. TTC repeats in Friedreich's ataxia. Proc Natl Acad Sci U S A. 2006; 103(31):11497-11502.

42. Grant L, Sun J, Xu H, Subramony SH, Chaires JB, Hebert MD. Rational selection of small molecules that increase transcription through the GAA repeats found in Friedreich's ataxia. FEBS Lett. 2006;580(22):5399-5405

43. Herman D, Jenssen K, Burnett R, Soragni E, Perlman SL, Gottesfeld JM. Histone deacetylase inhibitors reverse gene silencing in Friedreich's ataxia. Nat Chem Biol. 2006;2(10):551-558.

44. Sarsero JP, Li L, Wardan H, Sitte K, Williamson R, Ioannou PA. Upregulation of expression from the FRDA genomic locus for the therapy of Friedreich ataxia. J Gene Med. 2003;5(1):72-81.

45. Rai M, Soragni E, Chou CJ, et al. Two new pimelic diphenylamide HDAC inhibitors induce sustained frataxin upregulation in cells from Friedreich's ataxia patients and in a mouse model. PLoS One. 2010;5(1):e8825.

46. Xu C, Soragni E, Chou CJ, et al. Chemical probes identify a role for histone deacetylase 3 in Friedreich's ataxia gene silencing. Chem Biol. 2009;16(9):980-989.

47. Rai M, Soragni E, Jenssen K, et al. HDAC inhibitors correct frataxin deficiency in a Friedreich ataxia mouse model. PLoS One. 2008;3(4):e1958.

48. Gonzalez-Cabo P, Ros S, Palau F. Flavin adenine dinucleotide rescues the phenotype of frataxin deficiency. PLoS One. 2010;5(1):e8872.

49. Di Prospero NA, Sumner CJ, Penzak SR, Ravina B, Fischbeck KH, Taylor JP. Safety, tolerability, and pharmacokinetics of high-dose idebenone in patients with Friedreich ataxia. Arch Neurol. 2007;64(6): 803-808.

50. Meier T, Buyse G. Idebenone: an emerging therapy for Friedreich ataxia. J Neurol. 2009;256 Suppl 1:25-30.

51. Bodmer M, Vankan P, Dreier M, Kutz KW, Drewe J. Pharmacokinetics and metabolism of idebenone in healthy male subjects. Eur J Clin Pharmacol. 2009;65(5):493-501.

52. Schulz JB, Dehmer T, Schols L, et al. Oxidative stress in patients with Friedreich ataxia. Neurology. 2000;55(11):1719-1721.

53. Cooper JM, Schapira AH. Friedreich's ataxia: coenzyme Q10 and vitamin E therapy. Mitochondrion. 2007;7 Suppl:S127-S135.

54. Rustin P, Rotig A, Munnich A, Sidi D. Heart hypertrophy and function are improved by idebenone in Friedreich's ataxia. Free Radic Res. 2002;36(4):467-469.

55. Hausse AO, Aggoun Y, Bonnet D, et al. Idebenone and reduced cardiac hypertrophy in Friedreich's ataxia. Heart. 2002;87(4):346-349.

56. Mariotti C, Solari A, Torta D, Marano L, Fiorentini C, Di Donato S. Idebenone treatment in Friedreich patients: one-year-long randomized placebo-controlled trial. Neurology. 2003;60(10):1676-1679.

57. Di Prospero NA, Baker A, Jeffries N, Fischbeck KH. Neurological effects of high-dose idebenone in patients with Friedreich's ataxia: a randomised, placebo-controlled trial. Lancet Neurol. 2007;6(10):878-886.

58. Artuch R, Aracil A, Mas A, et al. Friedreich's ataxia: idebenone treatment in early stage patients. Neuropediatrics. 2002;33(4):190-193.

59. Pineda M, Arpa J, Montero R, et al. Idebenone treatment in paediatric and adult patients with Friedreich ataxia: long-term follow-up. Eur $J$ Paediatr Neurol. 2008;12(6):470-475.

60. Hart PE, Lodi R, Rajagopalan B, et al. Antioxidant treatment of patients with Friedreich ataxia: four-year follow-up. Arch Neurol. 2005;62(4):621-626.

61. Cooper JM, Korlipara LV, Hart PE, Bradley JL, Schapira AH. Coenzyme Q10 and vitamin E deficiency in Friedreich's ataxia: predictor of efficacy of vitamin E and coenzyme Q10 therapy. Eur J Neurol. 2008;15(12):1371-1379.

62. Pandolfo M. Drug insight: antioxidant therapy in inherited ataxias. Nat Clin Pract Neurol. 2008;4(2):86-96.

63. Orsucci D, Filosto M, Siciliano G, Mancuso M. Electron transfer mediators and other metabolites and cofactors in the treatment of mitochondrial dysfunction. Nutr Rev. 2009;67(8):427-438. 
64. Bjelakovic G, Nikolova D, Gluud LL, Simonetti RG, Gluud C. Mortality in randomized trials of antioxidant supplements for primary and secondary prevention: systematic review and meta-analysis. JAMA. 2007;297(8):842-857.

65. Schols L, Zange J, Abele M, et al. L-carnitine and creatine in Friedreich's ataxia. A randomized, placebo-controlled crossover trial. J Neural Transm. 2005;112(6):789-796.

66. Acquaviva F, Castaldo I, Filla A, et al. Recombinant human erythropoietin increases frataxin protein expression without increasing mRNA expression. Cerebellum. 2008;7(3):360-365.

67. Sturm B, Stupphann D, Kaun C, et al. Recombinant human erythropoietin: effects on frataxin expression in vitro. Eur J Clin Invest. 2005;35(11):711-717.

68. Siren AL, Fasshauer T, Bartels C, Ehrenreich H. Therapeutic potential of erythropoietin and its structural or functional variants in the nervous system. Neurotherapeutics. 2009;6(1):108-127.

69. Boesch S, Sturm B, Hering S, Goldenberg H, Poewe W, ScheiberMojdehkar B. Friedreich's ataxia: clinical pilot trial with recombinant human erythropoietin. Ann Neurol. 2007;62(5):521-524.

70. Boesch S, Sturm B, Hering S, et al. Neurological effects of recombinant human erythropoietin in Friedreich's ataxia: a clinical pilot trial. Mov Disord. 2008;23(13):1940-1944.

71. Finsterer J. Overview on visceral manifestations of mitochondrial disorders. Neth J Med. 2006;64(3):61-71.

72. Bensmail D, Quera Salva MA, Roche N, et al. Effect of intrathecal baclofen on sleep and respiratory function in patients with spasticity. Neurology. 2006;67(8):1432-1436.
73. Trouillas P, Serratrice G, Laplane D, et al. Levorotatory form of 5-hydroxytryptophan in Friedreich's ataxia. Results of a double-blind drug-placebo cooperative study. Arch Neurol. 1995;52(5):456-460.

74. Ristori G, Romano S, Visconti A, et al. Riluzole in cerebellar ataxia: a randomized, double-blind, placebo-controlled pilot trial. Neurology. 2010;74(10):839-845.

75. Ilg W, Synofzik M, Brotz D, Burkard S, Giese MA, Schols L. Intensive coordinative training improves motor performance in degenerative cerebellar disease. Neurology. 2009;73(22):1823-1830.

76. Maring JR, Croarkin E. Presentation and progression of Friedreich ataxia and implications for physical therapist examination. Phys Ther. 2007;87(12):1687-1696.

77. Fillyaw MJ, Ades PA. Endurance exercise training in Friedreich ataxia. Arch Phys Med Rehabil. 1989;70(10):786-788.

78. Goulipian C, Bensoussan L, Viton JM, Milhe-De Bovis V, Ramon J, Delarque A. Orthopedic shoes improve gait in Friedreich's ataxia: a clinical and quantified case study. Eur J Phys Rehabil Med. 2008;44(1): 93-98.

79. Delatycki MB, Holian A, Corben L, et al. Surgery for equinovarus deformity in Friedreich's ataxia improves mobility and independence. Clin Orthop Relat Res. 2005;(430):138-141.

80. Schulz JB, Boesch S, Burk K, et al. Diagnosis and treatment of Friedreich ataxia: a European perspective. Nat Rev Neurol. 2009;5(4):222-234.

81. Puccio H. Multicellular models of Friedreich ataxia. J Neurol. 2009;256 Suppl 1:18-24.
Neuropsychiatric Disease and Treatment

\section{Publish your work in this journal}

Neuropsychiatric Disease and Treatment is an international, peerreviewed journal of clinical therapeutics and pharmacology focusing on concise rapid reporting of clinical or pre-clinical studies on a range of neuropsychiatric and neurological disorders. This journal is indexed on PubMed Central, the 'PsycINFO' database and CAS, and is the official

\section{Dovepress}

journal of The International Neuropsychiatric Association (INA). The manuscript management system is completely online and includes a very quick and fair peer-review system, which is all easy to use. Visit http://www.dovepress.com/testimonials.php to read real quotes from published authors. 